\begin{tabular}{|c|c|}
\hline Title & Singular Bogoliubov transformations and inequival ent representations of canonical commutation relations \\
\hline Author(s) & A rai, A sao \\
\hline Citation & $\begin{array}{l}\text { Reviews in Mathematical Physics, 31(08), } 1950026 \\
\text { https://doi.org/10.1142/S0129055X 19500260 }\end{array}$ \\
\hline Issue Date & 2019-09 \\
\hline DOC URL & http:/hdl.handle.net/2115/79187 \\
\hline Rights & $\begin{array}{l}\text { Electronic version of an article published as Reviews in Mathematical Physics, Vol. 31, No. 08, } 1950026 \text { (2019), } \\
\text { https://doi.org/10.1142/S0129055X 19500260 @copyright W orld Scientific Publishing Company, } \\
\text { https:/www.worldscientific.com/worldscinet//mp }\end{array}$ \\
\hline Type & article (author version) \\
\hline File Information & RMP31-08_1950026.pdf \\
\hline
\end{tabular}

Instructions for use 


\title{
Singular Bogoliubov Transformations and Inequivalent Representations of Canonical Commutation Relations
}

\author{
Asao Arai \\ Department of Mathematics, Hokkaido University \\ Sapporo, Hokkaido 060-0810 \\ Japan \\ E-mail: arai@math.sci.hokudai.ac.jp
}

\begin{abstract}
We introduce a concept of singular Bogoliubov transformation on the abstract boson Fock space and construct a representation of canonical commutation relations (CCRs) which is inequivalent to any direct sum of the Fock representation. Sufficient conditions for the representation to be irreducible are formulated. Moreover, an example of such representations of CCRs is given.
\end{abstract}

Keywords: singular Bogoliubov transformation, canonical commutation relations, inequivalent representation, boson Fock space.

Mathematics Subject Classification 2010: 47L60.

\section{Introduction}

In this paper we introduce a concept of singular Bogoliubov transformation on the abstract boson Fock space over a Hilbert space $\mathscr{H}$. A singular Bogoliubov transformation is induced by a pair $(S, T)$ of unbounded linear operators on $\mathscr{H}$ obeying suitable constraints on their domain of definition and some consistency conditions (see (4.1) and (4.2)). As in the case of the standard bosonic Bogoliubov transformations (e.g., $[11,12,13]$ ), a singular Bogoliubov transformation also yields a representation $\pi_{B}(\mathscr{D})$ of the canonical commutation relations (CCRs) over a dense subspace $\mathscr{D}$ of $\mathscr{H}$. We are interested in conditions under which the representation $\pi_{B}(\mathscr{D})$ is inequivalent to any direct sum representation of the Fock representation $\pi_{\mathrm{F}}(\mathscr{D})$ of the CCRs over $\mathscr{D}$. We formulate also sufficient conditions for $\pi_{B}(\mathscr{D})$ to be irreducible.

One of the motivations for this work comes from representation theoretic considerations on the Casimir effect $[3,7,14]$. In the paper [5], there is a suggestion (a heuristic argument) that a representation of CCRs inequivalent to the Fock one may be associated with the Casimir effect and one may use this representation to explain the Casimir effect 
without invoking zero-point energy. But, in this paper, we do not discuss the Casimir effect.

The outline of the present paper is as follows. In Section 2, we recall the definition of representation of the CCRs over a complex inner product space and state a proposition on irreducibility of a representation of CCRs. In Section 3, we review the Fock representation of CCRs and prove some related facts. In Section 4, we introduce a singular Bogoliubov transformation. As mentioned above, it yields a representation $\pi_{B}(\mathscr{D})$ of the CCRs over $\mathscr{D}$. We prove that $\pi_{B}(\mathscr{D})$ is inequivalent to any direct sum representation of the Fock representation $\pi_{\mathrm{F}}(\mathscr{D})$ (Theorem 4.5 ). We formulate also sufficient conditions for $\pi_{B}(\mathscr{D})$ to be irreducible (Theorems 4.7 and 4.8). In Section 5, we show that, for each pair $(K, L)$ of injective symmetric operators with suitable properties, there may exist a singular Bogoliubov transformation and a representation $\pi_{K, L}(\mathscr{D})$ of the CCRs over $\mathscr{D}$, which is inequivalent to any direct sum representation of $\pi_{\mathrm{F}}(\mathscr{D})$ (Theorem 5.3). We show that, under an additional condition, $\pi_{K, L}(\mathscr{D})$ is irreducible (Theorem 5.4). In the last section, we discuss an example of $\pi_{K, L}(\mathscr{D})$ in the case $\mathscr{H}=L^{2}(\Lambda)$ with $\Lambda$ being a finite box in the $d$-dimensional Euclidean space $\mathbb{R}^{d}(d \geq 2)$. This example may be related to a Casimir effect which may be caused by a quantum scalar field on $\Lambda$. But, in the present paper, we do not discuss this aspect.

\section{Preliminary}

For a linear operator $A$ on a Hilbert space $\mathscr{X}$, we denote by $D(A)$ the domain of $A$. If $A$ is densely defined, then $A$ has its adjoint; we denote it by $A^{*}$.

Let $\mathscr{F}$ be a complex Hilbert space and $\mathscr{D}$ be a dense subspace of $\mathscr{F}$. Let $\mathscr{V}$ be a complex inner product space with inner product $\langle,\rangle_{\mathscr{V}}$ (linear in the second variable and anti-linear in the first) and norm $\|\cdot\|_{\mathscr{V}}$ (we sometimes omit the subscript $\mathscr{V}$ in $\langle,\rangle_{\mathscr{V}}$ and $\|\cdot\|_{\mathscr{V}}$ if there is no danger of confusion). Suppose that, for each $f \in \mathscr{V}$, a densely defined closed linear operator $C(f)$ on $\mathscr{F}$ is given. Then the triple $\left(\mathscr{F}, \mathscr{D},\left\{C(f), C(f)^{*} \mid f \in \mathscr{V}\right\}\right)$ is called a representation of the CCRs over $\mathscr{V}$ if the following (i)-(iii) hold:

(i) (domain invariance) For all $f \in \mathscr{V}, \mathscr{D} \subset D(C(f)) \cap D\left(C(f)^{*}\right), C(f) \mathscr{D} \subset \mathscr{D}$, $C(f)^{*} \mathscr{D} \subset \mathscr{D}$.

(ii) (anti-linearity in test vectors) For all $f, g \in \mathscr{V}$ and $\alpha, \beta \in \mathbb{C}$ (the set of complex numbers), $C(\alpha f+\beta g)=\alpha^{*} C(f)+\beta^{*} C(g)$ on $\mathscr{D}$, where, for $z \in \mathbb{C}, z^{*}$ denotes the complex conjugate of $z$.

(iii) (CCRs over $\mathscr{V}$ ) For all $f, g \in \mathscr{V}$,

$$
\left[C(f), C(g)^{*}\right]=\langle f, g\rangle_{\mathscr{V}}, \quad[C(f), C(g)]=0 \quad \text { on } \mathscr{D},
$$

where, for linear operators $A$ and $B$ on a Hilbert space, $[A, B]:=A B-B A$.

Two representations $\left(\mathscr{F}, \mathscr{D},\left\{C(f), C(f)^{*} \mid f \in \mathscr{V}\right\}\right)$ and $\left(\mathscr{F}^{\prime}, \mathscr{D}^{\prime},\left\{C^{\prime}(f), C^{\prime}(f)^{*} \mid f \in\right.\right.$ $\mathscr{V}\}$ ) of the CCRs over $\mathscr{V}$ are said to be equivalent if there exists a unitary operator $U: \mathscr{F} \rightarrow \mathscr{F}^{\prime}$ such that, for all $f \in \mathscr{V}, U C(f) U^{-1}=C^{\prime}(f)$. 
For two linear operators $A$ and $B$ on a Hilbert space $\mathscr{X}$, the symbol $A \subset B$ means that $B$ is an extension of $A$, i.e., $D(A) \subset D(B)$ and $A \Psi=B \Psi, \forall \Psi \in D(A)$.

As usual, we denote by $\mathfrak{B}(\mathscr{X})$ the Banach space of everywhere defined bounded linear operators on $\mathscr{X}$.

For a set $\mathfrak{A}$ of linear operators on a Hilbert space $\mathscr{X}$, the set

$$
\mathfrak{A}^{\prime}:=\{T \in \mathfrak{B}(\mathscr{X}) \mid T A \subset A T, \forall A \in \mathfrak{A}\} \subset \mathfrak{B}(\mathscr{X})
$$

is called the strong commutant of $\mathfrak{A}$.

The set $\mathfrak{A}$ is said to be reducible if there is a non-trivial closed subspace $\mathscr{M}$ of $\mathscr{X}$ $(\mathscr{M} \neq\{0\}, \mathscr{X})$ such that every $A \in \mathfrak{A}$ is reduced by $\mathscr{M}$ (i.e., $P_{\mathscr{M}} A \subset A P_{\mathscr{M}}$, where $P_{\mathscr{M}}$ is the orthogonal projection onto $\mathscr{M})$. The set $\mathfrak{A}$ is said to be irreducible if it is not reducible.

A representation $\left(\mathscr{F}, \mathscr{D},\left\{C(f), C(f)^{*} \mid f \in \mathscr{V}\right\}\right)$ of the CCRs over $\mathscr{V}$ is said to be reducible (resp. irreducible) if the set $\left\{C(f), C(f)^{*} \mid f \in \mathscr{V}\right\}$ is reducible (resp. irreducible).

The following fact is well known (see, e.g., [2, Proposition 5.9]):

Lemma 2.1 Let $\mathfrak{A}$ be a set of linear operators on $\mathscr{X}$.

(i) If $\mathfrak{A}^{\prime}=\mathbb{C} I:=\{\alpha I \mid \alpha \in \mathbb{C}\}$ (I denotes identity), then $\mathfrak{A}$ is irreducible.

(ii) If $\mathfrak{A}$ is an irreducible set of densely defined linear operators on $\mathscr{X}$ and $*$-invariant (i.e., $A \in \mathfrak{A} \Longrightarrow A^{*} \in \mathfrak{A}$ ), then $\mathfrak{A}^{\prime}=\mathbb{C} I$.

\section{Fock Representation of CCRs and Related Facts}

Let $\mathscr{H}$ be a complex Hilbert space and, for each $n \in \mathbb{N}$ (the set of natural numbers), $\otimes_{\mathrm{s}}^{n} \mathscr{H}$ be the $n$-fold symmetric tensor product Hilbert space of $\mathscr{H}$ with convention $\otimes_{\mathrm{s}}^{0} \mathscr{H}:=\mathbb{C}$. Then the boson Fock space over $\mathscr{H}$ is defined to be the direct sum Hilbert space of $\left\{\otimes_{\mathrm{s}}^{n} \mathscr{H}\right\}_{n=0}^{\infty}$ :

$$
\begin{aligned}
\mathscr{F}_{\mathrm{b}}(\mathscr{H}): & =\oplus_{n=0}^{\infty} \otimes_{\mathrm{s}}^{n} \mathscr{H} \\
& =\left\{\Psi=\left\{\Psi^{(n)}\right\}_{n=0}^{\infty} \mid \Psi^{(n)} \in \otimes_{\mathrm{s}}^{n} \mathscr{H}, n \geq 0, \sum_{n=0}^{\infty}\left\|\Psi^{(n)}\right\|^{2}<\infty\right\} .
\end{aligned}
$$

The subspace

$$
\begin{gathered}
\mathscr{F}_{0}(\mathscr{H}):=\left\{\Psi=\left\{\Psi^{(n)}\right\}_{n=0}^{\infty} \mid \Psi^{(n)} \in \otimes_{\mathrm{s}}^{n} \mathscr{H}, n \geq 0 \text { and there exists an } n_{0} \in \mathbb{N}\right. \text { such that } \\
\text { for all } \left.n \geq n_{0}, \Psi^{(n)}=0\right\}
\end{gathered}
$$

is dense in $\mathscr{F}_{\mathrm{b}}(\mathscr{H})$. This subspace is called the finite particle subspace of $\mathscr{F}_{\mathrm{b}}(\mathscr{H})$.

We denote by $A(f)$ the annihilation operator with test vector $f \in \mathscr{H}$ on $\mathscr{F}_{\mathrm{b}}(\mathscr{H})$, which is the unique densely defined closed linear operator on $\mathscr{F}_{\mathrm{b}}(\mathscr{H})$ such that its adjoint $A(f)^{*}$, called the creation operator with test vector $f$, takes the following form: for all $\Psi \in D\left(A(f)^{*}\right),\left(A(f)^{*} \Psi\right)^{(0)}=0$ and

$$
\left(A(f)^{*} \Psi\right)^{(n)}=\sqrt{n} S_{n}\left(f \otimes \Psi^{(n-1)}\right), \quad n \geq 1,
$$


where $S_{n}$ is the symmetrization operator on the $n$-fold tensor product $\otimes^{n} \mathscr{H}$ of $\mathscr{H}$. For all $f \in \mathscr{H}, \mathscr{F}_{0}(\mathscr{H}) \subset D(A(f)) \cap D\left(A(f)^{*}\right)$ and $A(f)$ and $A(f)^{*}$ leave $\mathscr{F}_{0}(\mathscr{H})$ invariant. Moreover, $\left\{A(f), A(f)^{*} \mid f \in \mathscr{H}\right\}$ satisfies the CCRs over $\mathscr{H}$ :

$$
\left[A(f), A(g)^{*}\right]=\langle f, g\rangle_{\mathscr{H}}, \quad[A(f), A(g)]=0, \quad\left[A(f)^{*}, A(g)^{*}\right]=0 \quad(f, g \in \mathscr{H})
$$

on $\mathscr{F}_{0}(\mathscr{H})$.

The following fact is well known (see, e.g., [2, Theorem 5.14]):

Theorem 3.1 Let $\mathscr{D}$ be a dense subspace of $\mathscr{H}$. Then $\left\{A(f), A(f)^{*} \mid f \in \mathscr{D}\right\}^{\prime}=\mathbb{C} I$ and

$$
\pi_{\mathrm{F}}(\mathscr{D}):=\left(\mathscr{F}_{\mathrm{b}}(\mathscr{H}), \mathscr{F}_{0}(\mathscr{H}),\left\{A(f), A(f)^{*} \mid f \in \mathscr{D}\right\}\right)
$$

is an irreducible representation of the CCRs over $\mathscr{D}$.

The representation $\pi_{\mathrm{F}}(\mathscr{D})$ is called the Fock representation of the CCRs over $\mathscr{D}$.

An anti-linear mapping $J: \mathscr{H} \rightarrow \mathscr{H}$ is called a conjugation on $\mathscr{H}$ if $J^{2}=I$ and $\|J f\|=\|f\|, f \in \mathscr{H}$.

The next proposition plays a basic role in the theory of representations of CCRs in boson Fock spaces:

Proposition 3.2 Assume that $\mathscr{H}$ is separable. Let $S$ and $T$ be (not necessarily bounded) linear operators on $\mathscr{H}$ with the following property: $D(S) \cap D(T)$ includes a dense subspace $\mathscr{D}$ of $\mathscr{H}$ such that $T \mathscr{D}$ is dense in $\mathscr{H}$ and $T_{\mathscr{D}}:=T \uparrow \mathscr{D}$, the restriction of $T$ to $\mathscr{D}$, is injective. Let $J$ be a conjugation on $\mathscr{H}$ and suppose that there exists a non-zero vector $\Omega \in \mathscr{F}_{\mathrm{b}}(\mathscr{H})$ such that, for all $\Psi \in \mathscr{F}_{0}(\mathscr{H})$ and $f \in \mathscr{D}$,

$$
\left\langle A(T f)^{*} \Psi, \Omega\right\rangle=-\langle A(J S f) \Psi, \Omega\rangle .
$$

Then $S T_{\mathscr{D}}^{-1}$ is a densely defined closable operator and its closure $\overline{S T_{\mathscr{D}}^{-1}}$ is Hilbert-Schmidt.

Proof. Let $\mathscr{D}_{T}:=T \mathscr{D}$. Then, by the present assumption, $\mathscr{D}_{T}$ is dense in $\mathscr{H}$. Hence $T_{\mathscr{D}}^{-1}$ is densely defined with $D\left(T_{\mathscr{D}}^{-1}\right)=\mathscr{D}_{T}$. By (3.3), we have $\left\langle A(g)^{*} \Psi, \Omega\right\rangle=$ $-\left\langle A\left(J S T_{\mathscr{D}}^{-1} g\right) \Psi, \Omega\right\rangle$ for all $g \in \mathscr{D}_{T}$. Hence it follows from [1, Proposition 3.3 $]^{1}$ that $S T_{\mathscr{D}}^{-1}$ is bounded (hence closable) and $\overline{S T_{\mathscr{D}}^{-1}}$ is Hilbert-Schmidt.

Lemma 3.3 Let $X$ and $Y$ be (not necessarily bounded) linear operators on $\mathscr{H}$ such that there exists a dense subspace $\mathscr{D} \subset D(X) \cap D(Y)$ and the following equation holds:

$$
\langle X f, X g\rangle-\langle Y f, Y g\rangle=\langle f, g\rangle, \quad f, g \in \mathscr{D} .
$$

Let $X_{\mathscr{D}}:=X \uparrow \mathscr{D}$. Then $X_{\mathscr{D}}$ is injective and $X_{\mathscr{D}}^{-1}$ is bounded with $\left\|X_{\mathscr{D}}^{-1}\right\| \leq 1$.

\footnotetext{
${ }^{1}$ In the cited proposition, it is assumed that an operator $L$ satisfying $\left\langle A(g)^{*} \Psi, \Omega\right\rangle=\langle A(J L g) \Psi, \Omega\rangle$ is bounded. But, as is seen from the proof, it is not necessary.
} 
Proof. It follows from (3.4) that, for all $f \in \mathscr{D},\left\|X_{\mathscr{D}} f\right\|^{2}=\|f\|^{2}+\|Y f\|^{2} \geq\|f\|^{2}$, which implies the desired result.

The following proposition is new (to the author's best knowledge) and plays a crucial role in the present paper.

Proposition 3.4 Assume that $\mathscr{H}$ is separable. Let $X$ and $Y$ be as in Lemma 3.3 and suppose that $X$ is unbounded and $X \mathscr{D}$ is dense in $\mathscr{H}$. Let $\Omega$ be a vector in $\mathscr{F}_{\mathrm{b}}(\mathscr{H})$ satisfying

$$
\left\langle A(X f)^{*} \Psi, \Omega\right\rangle=-\langle A(J Y f) \Psi, \Omega\rangle, \quad \Psi \in \mathscr{F}_{0}(\mathscr{H}), f \in \mathscr{D} .
$$

Then $\Omega=0$.

Proof. We prove the proposition by reductio ad absurdum. Suppose that there existed a non-zero vector $\Omega \in \mathscr{F}_{\mathrm{b}}(\mathscr{H})$ such that (3.5) holds. Since $\operatorname{Ran} X_{\mathscr{D}}$ is dense by the present assumption and $X_{\mathscr{D}}$ is injective by Lemma 3.3, it follows from Proposition 3.2 that $K:=\overline{Y X_{\mathscr{D}}^{-1}}$ is Hilbert-Schmidt. Hence $K^{*} K$ is trace class. Using (3.4) and a limiting argument, one can show that

$$
K^{*} K=I-L^{*} L,
$$

where $L:=\overline{X_{\mathscr{D}}^{-1}}$. For a linear operator $A$, we denote by $\sigma(A)$ the spectrum of $A$. Since $K^{*} K$ is a non-negative trace class operator, it is a non-negative compact operator. Hence $\sigma\left(K^{*} K\right) \backslash\{0\}=\left\{\lambda_{n}\right\}_{n=1}^{N}$, where $N<\infty$ or $N=\infty$ and $\lambda_{n}$ is a positive eigenvalue of $K^{*} K$ with a finite multiplicity; if $N=\infty$, then $\lim _{n \rightarrow \infty} \lambda_{n}=0 \cdots(*)$. Hence $\sigma\left(L^{*} L\right) \backslash\{1\}=$ $\left\{1-\lambda_{n}\right\}_{n=1}^{N}$.

Suppose that there existed a constant $\gamma>0$ such that $L^{*} L \geq \gamma$. Then, for all $g \in \operatorname{Ran} X_{\mathscr{D}},\left\|X_{\mathscr{D}}^{-1} g\right\|^{2} \geq \gamma\|g\|^{2}$. Hence $\left\|X_{\mathscr{D}} f\right\|^{2} \leq \gamma^{-1}\|f\|^{2}, f \in \mathscr{D}$. But this contradicts the unboundedness of $X$. Hence $\inf \sigma\left(L^{*} L\right)=0$. Hence $N=\infty$ and there exists a subsequence $\left\{\lambda_{n_{k}}\right\}_{k=1}^{\infty}$ such that $\lim _{k \rightarrow \infty}\left(1-\lambda_{n_{k}}\right)=0$, i.e., $\lim _{k \rightarrow \infty} \lambda_{n_{k}}=1$. But this contradicts $(*)$. Thus we arrive at a contradiction.

In concluding this section, we state a fact which may be used to prove the irreducibility of representations of CCRs. For each $f \in \mathscr{H}$, one has a self-adjoint operator

$$
\Phi(f):=\frac{1}{\sqrt{2}} \overline{\left(A(f)^{*}+A(f)\right)}
$$

called the Segal field operator with test vector $f$. It is easy to see that $\Phi(f)$ leaves $\mathscr{F}_{0}(\mathscr{H})$ invariant and, for all $f, g \in \mathscr{H}$,

$$
[\Phi(f), \Phi(g)]=i \operatorname{Im}\langle f, g\rangle \quad \text { on } \mathscr{F}_{0}(\mathscr{H}),
$$

where, for a complex number $z \in \mathbb{C}, \operatorname{Im} z$ denotes the imaginary part of $z$.

Let $J$ be a conjugation on $\mathscr{H}$. Then the set

$$
\mathscr{H}_{J}:=\{f \in \mathscr{H} \mid J f=f\}
$$

is a real Hilbert space with the inner product of $\mathscr{H}$. It follows that

$$
\mathscr{H}=\left\{f+i g \mid f, g \in \mathscr{H}_{J}\right\} .
$$

Let $A$ and $B$ be densely defined linear operators on $\mathscr{H}$ with the following properties (i) and (ii): 
(i) $J A \subset A J, J B \subset B J$.

(ii) There exists a subspace $\mathscr{E}$ in $\mathscr{H}_{J}$ such that $\mathscr{E} \subset D(A) \cap D(B)$ and, for all $f, g \in \mathscr{E}$, $\langle A f, B g\rangle=\langle f, g\rangle$.

Property (i) means that, for all $\Psi \in D(A), J \Psi \in D(A)$ and $A J \Psi=J A \Psi$. Hence, in particular, $A\left(D(A) \cap \mathscr{H}_{J}\right) \subset \mathscr{H}_{J}$. Similarly one has that $B\left(D(B) \cap \mathscr{H}_{J}\right) \subset \mathscr{H}_{J}$.

Let

$$
\phi_{A}(f):=\Phi(A f), \quad \pi_{B}(f):=\Phi(i B g), \quad f \in D(A) \cap \mathscr{H}_{J}, g \in D(B) \cap \mathscr{H}_{J} .
$$

Then it follows from (3.7) that $\phi_{A}(f)$ and $\pi_{B}(g)$ obey the Heisenberg commutation relations over $\mathscr{E}$ on $\mathscr{F}_{0}(\mathscr{H})$ : for all $f, g \in \mathscr{E}$,

$$
\left[\phi_{A}(f), \pi_{B}(g)\right]=i\langle f, g\rangle, \quad\left[\phi_{A}(f), \phi_{A}(g)\right]=0, \quad\left[\pi_{B}(f), \pi_{B}(g)\right]=0
$$

on $\mathscr{F}_{0}(\mathscr{H})$. In fact, $e^{i \phi_{A}(f)}$ and $e^{i \pi_{B}(g)}$ obey the Weyl relations (see, e.g., [2, Theorem $5.25(\mathrm{ii})])$ :

$$
e^{i \phi_{A}(f)} e^{i \pi_{B}(g)}=e^{-i\langle f, g\rangle} e^{i \pi_{B}(g)} e^{i \phi_{A}(f)}, \quad f, g \in \mathscr{E} .
$$

Lemma 3.5 Let $C:=A+i B$. Suppose that $C \mathscr{E}$ is dense in $\mathscr{H}$. Then $\left\{e^{i \phi_{A}(f)}, e^{i \pi_{B}(f)} \mid f \in\right.$ $\mathscr{E}\}^{\prime}=\mathbb{C} I$. In particular, $\left\{e^{i \phi_{A}(f)}, e^{i \pi_{B}(f)} \mid f \in \mathscr{E}\right\}$ is irreducible.

Proof. Let $T \in\left\{e^{i \phi_{A}(f)}, e^{i \pi_{B}(f)} \mid f \in \mathscr{E}\right\}^{\prime}$. Then, for all $f \in \mathscr{E}, T e^{i \phi_{A}(f)}=e^{i \phi_{A}(f)} T$ and $T e^{i \pi_{B}(f)}=e^{i \pi_{B}(f)} T$. By a general formula for the Weyl operator $e^{i \Phi(u)}(u \in \mathscr{H})$ (see, e.g., $[2$, Theorem 5.25(ii)]), we have

$$
e^{i \Phi(C f)}=e^{i\|f\|^{2} / 2} e^{i \phi_{A}(f)} e^{i \pi_{B}(f)}, \quad f \in \mathscr{E} .
$$

Hence it follows that $T e^{i \Phi(C f)}=e^{i \Phi(C f)} T, f \in \mathscr{E}$. Recall that, for any dense subspace $\mathscr{D}$ of $\mathscr{H},\left\{e^{i \Phi(u)} \mid u \in \mathscr{D}\right\}$ is irreducible (e.g., [2, Theorem 5.25(iv)]). Note that $\left\{e^{i \Phi(u)} \mid u \in \mathscr{D}\right\}$ is $*$-invariant. Hence, by Lemma 2.1(ii), $\left\{e^{i \Phi(u)} \mid u \in \mathscr{D}\right\}^{\prime}=\mathbb{C} I$. Therefore $\left\{e^{i \Phi(C f)} \mid f \in\right.$ $\mathscr{E}\}^{\prime}=\mathbb{C} I$. Hence $T=\alpha I$ for some $\alpha \in \mathbb{C}$.

\section{Singular Bogoliubov Transformations and Repre- sentations of CCRs}

\subsection{Definitions}

Let $T$ and $S$ be densely defined (not necessarily bounded) linear operators on $\mathscr{H}$ such that there exists a dense subspace $\mathscr{D} \subset D(T) \cap D(S)$ and the following equations hold:

$$
\begin{aligned}
& \langle T f, T g\rangle-\langle S f, S g\rangle=\langle f, g\rangle, \\
& \langle T f, J S g\rangle=\langle S f, J T g\rangle, \quad f, g \in \mathscr{D},
\end{aligned}
$$

where $J$ is a conjugation on $\mathscr{H}$. 
For each $f \in \mathscr{D}$, the operator

$$
B_{0}(f):=A(T f)+A(J S f)^{*}
$$

is a densely defined linear operator with $D\left(B_{0}(f)\right) \supset \mathscr{F}_{0}(\mathscr{H})$ and $B_{0}(f)^{*}$ is densely defined with $D\left(B_{0}(f)^{*}\right) \supset \mathscr{F}_{0}(\mathscr{H})$. Hence $B_{0}(f)$ is closable. Therefore one can define a densely defined closed operator $B(f)$ on $\mathscr{F}_{\mathrm{b}}(\mathscr{H})$ by

$$
B(f):=\overline{B_{0}(f)}
$$

It is obvious that $B(f)$ and $B(f)^{*}$ leave $\mathscr{F}_{0}(\mathscr{H})$ invariant. Moreover, using $(4.1)$ and $(4.2)$, one can easily show that $B(\cdot)$ and $B(\cdot)^{*}$ satisfy the CCRs over $\mathscr{D}$ on $\mathscr{F}_{0}(\mathscr{H})$ : for all $f, g \in \mathscr{D}$,

$$
\left[B(f), B(g)^{*}\right]=\langle f, g\rangle, \quad[B(f), B(g)]=0, \quad\left[B(f)^{*}, B(g)^{*}\right]=0 \quad \text { on } \mathscr{F}_{0}(\mathscr{H}) .
$$

Therefore

$$
\pi_{B}(\mathscr{D}):=\left(\mathscr{F}_{\mathrm{b}}(\mathscr{H}), \mathscr{F}_{0}(\mathscr{H}),\left\{B(f), B(f)^{*} \mid f \in \mathscr{D}\right\}\right.
$$

is a representation of the CCRs over $\mathscr{D}$.

The correspondence $T_{B}:\left(A(\cdot), A(\cdot)^{*}\right) \mapsto\left(B(\cdot), B(\cdot)^{*}\right)$ is a generalization of the standard bosonic Bogoliubov transformation (see, e.g., [11, 12, 13], [9, p.316], [6, §2] and references therein) in the sense that $S$ or $T$ may be unbounded and $T_{B}$ is not necessarily invertible. Equation (4.1) implies that $T$ is bounded if and only if $S$ is bounded. Based on this property, we say that the Bogoliubov transformation $T_{B}$ is singular if $T$ or $S$ is unbounded (then both $T$ and $S$ are unbounded).

\subsection{Inequivalence to any direct sum representation of the Fock representation $\pi_{\mathrm{F}}(\mathscr{D})$}

We want to find conditions under which $\pi_{B}(\mathscr{D})$ is inequivalent to any direct sum representation of the Fock representation $\pi_{\mathrm{F}}(\mathscr{D})$.

Lemma 4.1 Let $\mathscr{D}$ be a dense subspace of $\mathscr{H}$ and $\pi_{C}(\mathscr{D}):=\left(\mathscr{F}, \mathscr{D},\left\{C(f), C(f)^{*} \mid f \in\right.\right.$ $\mathscr{D}\})$ be a representation of the CCRs over $\mathscr{D}$. Suppose that $\pi_{C}(\mathscr{D})$ is equivalent to a direct sum representation $\oplus_{n=1}^{N} \pi_{\mathrm{F}}(\mathscr{D})$ of $\pi_{\mathrm{F}}(\mathscr{D})$ with $N<\infty$ or $N=\infty$. Then there exists a non-zero vector $\Omega \in \cap_{f \in \mathscr{D}} D(C(f))$ such that $C(f) \Omega=0, f \in \mathscr{D}$.

Proof. By the present assumption, there exists a unitary operator $U$ from $\mathscr{F}_{\mathrm{b}}(\mathscr{H})$ to $\mathscr{G}_{N}:=\oplus_{n=1}^{N} \mathscr{F}_{\mathrm{b}}(\mathscr{H})$ such that $U C(f) U^{-1}=\oplus_{n=1}^{N} A(f), f \in \mathscr{D}$. Let $\Omega_{\mathscr{H}}$ be the Fock vacuum in $\mathscr{F}_{\mathrm{b}}(\mathscr{H})$ :

$$
\Omega_{\mathscr{H}}^{(0)}=1, \quad \Omega_{\mathscr{H}}^{(n)}=0, \quad n \geq 1 .
$$

Then one has

$$
A(f) \Omega_{\mathscr{H}}=0, \quad f \in \mathscr{H} .
$$


It is easy to see that $\Psi_{0}:=\oplus_{n=1}^{N} n^{-1} \Omega_{\mathscr{H}}$ is a non-zero vector in $\mathscr{G}_{N}$ (note that, in the case $\left.N=\infty, \sum_{n=1}^{\infty}\left\|n^{-1} \Omega_{\mathscr{H}}\right\|^{2}=\sum_{n=1}^{\infty} n^{-2}<\infty\right)$. By (4.6), $\Psi_{0} \in D\left(\oplus_{n=1}^{N} A(f)\right), f \in \mathscr{D}$ and $\left(\oplus_{n=1}^{N} A(f)\right) \Psi_{0}=0$. Hence, putting $\Omega:=U^{-1} \Psi_{0}$, one sees that $\Omega \in \cap_{f \in \mathscr{D}} D(C(f))$ and $C(f) \Omega=0, f \in \mathscr{D}$.

To state and prove results on the above mentioned inequivalence, we consider two cases separately: the one is the case where $T$ is bounded and the other is the case where $T$ is unbounded.

Lemma 4.2 Assume that $\mathscr{H}$ is separable. Suppose that $T$ is bounded with $T \mathscr{D}$ being dense in $\mathscr{H}$ and $\bar{S}$ is not Hilbert-Schmidt. Then there exist no non-zero vectors $\Omega \in$ $\cap_{f \in \mathscr{D}} D(B(f))$ such that $B(f) \Omega=0, f \in \mathscr{D}$.

Proof. Suppose that there existed a non-zero vector $\Omega \in \cap_{f \in \mathscr{D}} D(B(f))$ that $B(f) \Omega=$ $0, f \in \mathscr{D}$. Then, for all $\Psi \in \mathscr{F}_{0}(\mathscr{H})$ and $f \in \mathscr{D},(3.3)$ holds. Hence, by Proposition 3.2, $\overline{S T_{\mathscr{D}}^{-1}}$ is Hilbert-Schmidt. By the present assumption, $\overline{T_{\mathscr{D}}}=\bar{T}$ is bounded. Hence $U:=\overline{S T_{\mathscr{D}}^{-1}} \overline{T_{\mathscr{D}}}$ is Hilbert-Schmidt. One has $U=\bar{S}$. Hence $\bar{S}$ is Hilbert-Schmidt. But this contradicts the assumption that $\bar{S}$ is not Hilbert-Schmidt.

Theorem 4.3 Assume that $\mathscr{H}$ is separable. Suppose that $T$ is bounded with $T \mathscr{D}$ being dense in $\mathscr{H}$ and $\bar{S}$ is not Hilbert-Schmidt. Then $\pi_{B}(\mathscr{D})$ is inequivalent to any direct sum representations of the Fock representation $\pi_{\mathrm{F}}(\mathscr{D})$. In particular, if $\pi_{B}(\mathscr{D})$ is irreducible, then $\pi_{B}(\mathscr{D})$ is inequivalent to $\pi_{\mathrm{F}}(\mathscr{D})$.

Proof. Suppose that $\pi_{B}(\mathscr{D})$ were equivalent to a direct sum representation of $\pi_{\mathrm{F}}(\mathscr{D})$. Then, by Lemma 4.1, there exists a non-zero vector $\Omega \in \cap_{f \in \mathscr{D}} D(B(f))$ such that $B(f) \Omega=$ $0, f \in \mathscr{D}$. But this contradicts Lemma 4.2.

We next consider the case where $T$ is unbounded.

Lemma 4.4 Assume that $\mathscr{H}$ is separable. Suppose that $T$ is unbounded and $T \mathscr{D}$ is dense in $\mathscr{H}$. Then there exist no non-zero vectors $\Omega \in \cap_{f \in \mathscr{D}} D(B(f))$ such that $B(f) \Omega=0, f \in$ $\mathscr{D}$.

Proof. Suppose that there existed a non-zero vector $\Omega \in \cap_{f \in \mathscr{D}} D(B(f))$ such that $B(f) \Omega=0, f \in \mathscr{D}$. Then (3.5) holds with $X=T$ and $Y=S$. But this contradicts Proposition 3.4.

The following theorem is one of the main results in the abstract framework of the present paper.

Theorem 4.5 Assume that $\mathscr{H}$ is separable. Suppose that $T$ is unbounded and $T \mathscr{D}$ is dense in $\mathscr{H}$. Then $\pi_{B}(\mathscr{D})$ is inequivalent to any direct sum representation of the Fock representation $\pi_{\mathrm{F}}(\mathscr{D})$. In particular, if $\pi_{B}(\mathscr{D})$ is irreducible, then $\pi_{B}(\mathscr{D})$ is inequivalent to $\pi_{\mathrm{F}}(\mathscr{D})$.

Proof. Suppose that $\pi_{B}(\mathscr{D})$ were equivalent to a direct sum representation of $\pi_{\mathrm{F}}(\mathscr{D})$. Then, by Lemma 4.1, there exists a non-zero vector $\Omega \in \cap_{f \in \mathscr{D}} D(B(f))$ such that $B(f) \Omega=$ $0, f \in \mathscr{D}$. But this contradicts Lemma 4.4. 


\subsection{Irreducibility}

In this subsection, we formulate sufficient conditions for $\pi_{B}(\mathscr{D})$ to be irreducible. To be as general as possible, we consider two cases. The first one uses the following condition:

Assumption (I) There exists a dense subspace $\mathscr{D}_{1}$ of $\mathscr{H}$ such that the following (i) and (ii) hold:

(i) $\mathscr{D}_{1} \subset D\left(T T^{*}\right) \cap D\left(J S S^{*} J\right) \cap D\left(S T^{*}\right) \cap D\left(J T S^{*} J\right)$.

(ii) $T^{*} \mathscr{D}_{1} \subset \mathscr{D},\left(S^{*} J\right) \mathscr{D}_{1} \subset \mathscr{D}$ and

$$
T T^{*}-J S S^{*} J=I, \quad S T^{*} J=J T S^{*} \text { on } \mathscr{D}_{1} .
$$

Lemma 4.6 Suppose that Assumption (I) holds. Then, for all $f \in \mathscr{D}_{1}$,

$$
A(f)=B\left(T^{*} f\right)-B\left(S^{*} J f\right)^{*} \text { on } \mathscr{F}_{0}(\mathscr{H}) .
$$

Proof. One needs only to compute the right hand side of (4.7) using (4.3) and Assumption (I).

Theorem 4.7 Suppose in addition to (4.1) and (4.2) that Assumption (I) holds. Then $\pi_{B}(\mathscr{D})$ is irreducible.

Proof. Let $Z \in\left\{B(f), B(f)^{*} \mid f \in \mathscr{D}\right\}^{\prime}$. Then $Z \in \mathfrak{B}\left(\mathscr{F}_{\mathrm{b}}(\mathscr{H})\right)$ and for all $f \in \mathscr{D}$, $Z B(f) \subset B(f) Z, Z B(f)^{*} \subset B(f)^{*} Z$. By using (4.7), one can show that, for all $\Phi, \Psi \in$ $\mathscr{F}_{0}(\mathscr{H})$ and $f \in \mathscr{D}_{1}$,

$$
\langle\Phi, Z A(f) \Psi\rangle=\left\langle A(f)^{*} \Phi, Z \Psi\right\rangle .
$$

Since $\mathscr{F}_{0}(\mathscr{H})$ is a core for $A(f)^{*}$, it follows that $Z \Psi \in D\left(A(f)^{* *}\right)=D(A(f))$ and $A(f) Z \Psi=Z A(f) \Psi$. Since $\mathscr{F}_{0}(\mathscr{H})$ is a core for $A(f)$, this implies that $Z A(f) \subset A(f) Z$. Similarly one has $Z A(f)^{*} \subset A(f)^{*} Z$ for all $f \in \mathscr{D}_{1}$. Hence $Z \in\left\{A(f), A(f)^{*} \mid f \in \mathscr{D}_{1}\right\}^{\prime}$. By Theorem 3.1, $\left\{A(f), A(f)^{*} \mid f \in \mathscr{D}_{1}\right\}^{\prime}=\mathbb{C} I$. Hence $\left\{B(f), B(f)^{*} \mid f \in \mathscr{D}\right\}^{\prime}=\mathbb{C} I$. Therefore, by Lemma 2.1(i), $\left\{B(f), B(f)^{*} \mid f \in \mathscr{D}\right\}$ is irreducible.

Another criterion for the irreducibility of $\pi_{B}(\mathscr{D})$ is given as follows:

Theorem 4.8 Suppose in addition to (4.1) and (4.2) that

$$
J S \subset S J, \quad J T \subset T J .
$$

Let $\mathscr{D}_{J}:=\mathscr{D} \cap \mathscr{H}_{J}, R:=(1+i) T+(1-i) S$ and suppose that $R \mathscr{D}_{J}$ is dense in $\mathscr{H}$. Then $\pi_{B}(\mathscr{D})$ is irreducible.

Proof. Let $Z \in\left\{B(f), B(f)^{*} \mid f \in \mathscr{D}\right\}^{\prime}$. Then it is easy to see that, for all $f \in \mathscr{D}_{J}$,

$$
\frac{1}{\sqrt{2}}\left(B(f)^{*}+B(f)\right)=\phi_{T+S}(f), \quad \frac{i}{\sqrt{2}}\left(B(f)^{*}-B(f)\right)=\pi_{T-S}(f)
$$

on $\mathscr{F}_{0}(\mathscr{H})$. Hence, in the same way as in the proof of Theorem 4.8, one can show that $Z \phi_{T+S}(f) \subset \phi_{T+S}(f) Z$ and $Z \pi_{T-S}(f) \subset \pi_{T-S}(f) Z$. Since $\phi_{T+S}(f)$ and $\pi_{T-S}(f)$ are selfadjoint, it follows that $Z e^{i \phi_{T+S}(f)}=e^{i \phi_{T+S}(f)} Z$ and $Z e^{i \pi_{T-S}(f)}=e^{i \pi_{T-S}(f)} Z$. Note that $T+S+i(T-S)=R$. Hence, by Lemma 3.5, $Z=\alpha I$ for some $\alpha \in \mathbb{C}$. Thus $\pi_{B}(\mathscr{D})$ is irreducible. 


\section{A General Class of Singular Bogoliubov Transfor- mations}

In this section, we introduce a general class of singular Bogoliubov transformations. Let $K$ and $L$ be injective (not necessarily bounded) symmetric operators on a Hilbert space $\mathscr{H}$ such that

$$
\mathscr{D}_{K, L}:=D\left(K^{-1} L\right) \cap D\left(K L^{-1}\right)
$$

is dense in $\mathscr{H}$ and, for a conjugation $J$ on $\mathscr{H}$,

$$
J K \subset K J, \quad J L \subset L J .
$$

Then one can define densely defined linear operators

$$
T_{ \pm}:=\frac{1}{2}\left(K^{-1} L \pm K L^{-1}\right)
$$

with $D\left(T_{ \pm}\right)=\mathscr{D}_{K, L}$. It follows from (5.2) that

$$
J T_{ \pm} \subset T_{ \pm} J
$$

Lemma 5.1 For all $f, g \in \mathscr{D}_{K, L}$,

$$
\begin{aligned}
& \left\langle T_{+} f, T_{+} g\right\rangle-\left\langle T_{-} f, T_{-} g\right\rangle=\langle f, g\rangle, \\
& \left\langle T_{+} f, J T_{-} g\right\rangle=\left\langle T_{-} f, J T_{+} g\right\rangle .
\end{aligned}
$$

Proof. This follows from direct computations, where the symmetry of $K$ and $L$ are used.

Remark 5.2 Operators of type $T_{ \pm}$with $K$ and $L$ being injective self-adjoint operators such that $K^{-1} L$ and $K L^{-1}$ are bounded are introduced in [1, Lemma 5.8]. But here we are mainly interested in the case where $T_{ \pm}$are unbounded.

Let $\mathscr{D}$ be a dense subspace of $\mathscr{H}$ such that $\mathscr{D} \subset \mathscr{D}_{K, L}$. Then, as in Section 4, one can define a densely defined closed linear operator

$$
B_{K, L}(f):=\overline{A\left(T_{+} f\right)+A\left(J T_{-} f\right)^{*}}, \quad f \in \mathscr{D}
$$

and see that

$$
\pi_{K, L}(\mathscr{D}):=\left(\mathscr{F}_{\mathrm{b}}(\mathscr{H}), \mathscr{F}_{0}(\mathscr{H}),\left\{B_{K, L}(f), B_{K, L}(f)^{*} \mid f \in \mathscr{D}\right\}\right.
$$

is a representation of the CCRs over $\mathscr{D}$.

Note that, if $T_{+}$or $T_{-}$is unbounded, then the correspondence: $\left(A(\cdot), A(\cdot)^{*}\right) \mapsto$ $\left(B_{K, L}(\cdot), B_{K, L}(\cdot)^{*}\right)$ is a singular Bogoliubov transformation. Thus, for each pair $(K, L)$ of injective symmetric operators with additional properties, there exists a singular Bogoliubov transformation.

A simple application of Lemma 4.4 and Theorem 4.5 yields the following theorem: 
Theorem 5.3 Let $\mathscr{H}$ be separable. Suppose that $T_{+}$is unbounded and $T_{+} \mathscr{D}$ is dense. Then:

(i) There exists no non-zero vector $\Omega \in \cap_{f \in \mathscr{D}} D\left(B_{K, L}(f)\right)$ such that $B_{K, L}(f) \Omega=$ $0, f \in \mathscr{D}$.

(ii) The representation $\pi_{K, L}(\mathscr{D})$ is inequivalent to any direct sum representation of the Fock representation $\pi_{\mathrm{F}}(\mathscr{D})$. In particular, if $\pi_{K, L}(\mathscr{D})$ is irreducible, then $\pi_{K, L}(\mathscr{D})$ is inequivalent to $\pi_{\mathrm{F}}(\mathscr{D})$.

With regard to irreducibility of $\pi_{K, L}(\mathscr{D})$, we have the following result.

Theorem 5.4 Suppose that $\left(K^{-1} L+i K L^{-1}\right)\left(\mathscr{D} \cap \mathscr{H}_{J}\right)$ is dense in $\mathscr{H}$. Then $\pi_{K, L}(\mathscr{D})$ is irreducible.

Proof. We need only to apply Theorem 4.8 to the case where $T=T_{+}, S=T_{-}$. In this case $R=K^{-1} L+i K L^{-1}$.

\section{An Example}

In this section we show by an explicit construction that there exists a pair $(K, L)$ of symmetric operators and a conjugation $J$, satisfying conditions described in Section 5 so that the conclusions of Theorems 5.3 and 5.4 hold.

The example given below is related to a construction of two quantum scalar fields on the finite box

$$
\Lambda:=(0, L)^{d-1} \times\left(0, L_{d}\right)=\left\{\boldsymbol{x}=\left(x_{1}, \ldots, x_{d}\right) \mid x_{1}, \ldots, x_{d-1} \in(0, L), x_{d} \in\left(0, L_{d}\right)\right\}
$$

in the $d$-dimensional Euclidean space $\mathbb{R}^{d}$ with $d \geq 2, L>0$ and $L_{d}>0$.

Remark 6.1 There may be another choice of $\Lambda$. For example $\Lambda=\mathbb{R}^{d-1} \times\left(0, L_{d}\right)$. But, in this paper, we restrict ourselves to the case given above.

We denote by $\Delta_{\mathrm{D}}$ the Dirichlet Laplacian for $\Lambda$ on $L^{2}(\Lambda)$ (see, e.g., [10, p.263] for the definition of $\left.\Delta_{\mathrm{D}}\right){ }^{2}$ The operator $-\Delta_{\mathrm{D}}$ is a non-negative self-adjoint operator. Let

$$
\Gamma:=\left\{\boldsymbol{k}=\left(k_{1}, \ldots, k_{d}\right) \mid k_{j} \in \frac{\pi}{L} \mathbb{N}, j=1, \ldots, d-1, k_{d} \in \frac{\pi}{L_{d}} \mathbb{N}\right\}
$$

where $\alpha \mathbb{N}:=\{\alpha n \mid n \in \mathbb{N}\}(\alpha \in \mathbb{R})$. It is well known that the spectrum $\sigma\left(-\Delta_{\mathrm{D}}\right)$ of $-\Delta_{\mathrm{D}}$ is purely discrete with

$$
\sigma\left(-\Delta_{\mathrm{D}}\right)=\left\{\boldsymbol{k}^{2} \mid \boldsymbol{k} \in \Gamma\right\}
$$

\footnotetext{
${ }^{2}$ One can consider also Laplacians with other boundary conditions. But, in the present paper, we treat only the Dirichlet Laplacian for simplicity.
} 
An eigenvector of $-\Delta_{\mathrm{D}}$ with eigenvalue $\boldsymbol{k}^{2}$ is given by

$$
\varphi_{\boldsymbol{k}}(\boldsymbol{x}):=\prod_{j=1}^{d} \varphi_{k_{j}}\left(x_{j}\right), \quad \boldsymbol{x}=\left(x_{1}, \ldots, x_{d}\right) \in \Lambda
$$

with

$$
\begin{aligned}
\varphi_{k_{j}}\left(x_{j}\right) & :=\sqrt{\frac{2}{L}} \sin \left(k_{j} x_{j}\right), \quad x_{j} \in(0, L), \quad j=1, \ldots, d-1, \\
\varphi_{k_{d}}\left(x_{d}\right) & :=\sqrt{\frac{2}{L_{d}}} \sin \left(k_{d} x_{d}\right), \quad x_{d} \in\left(0, L_{d}\right) .
\end{aligned}
$$

It is well known that $\left\{\varphi_{\boldsymbol{k}} \mid \boldsymbol{k} \in \Gamma\right\}$ is a complete orthonormal system (CONS) of $L^{2}(\Lambda)$.

Let $m \geq 0$ be a constant and

$$
h:=\left(-\Delta_{\mathrm{D}}+m^{2}\right)^{1 / 2}
$$

Then we have

$$
h \varphi_{\boldsymbol{k}}=\omega(\boldsymbol{k}) \varphi_{\boldsymbol{k}}, \quad \boldsymbol{k} \in \Gamma,
$$

with

$$
\omega(\boldsymbol{k}):=\sqrt{\boldsymbol{k}^{2}+m^{2}} .
$$

Hence the spectrum of $h$ is purely discrete with

$$
\sigma(h)=\{\omega(\boldsymbol{k}) \mid \boldsymbol{k} \in \Gamma\} .
$$

Note that

$$
0<\omega(\boldsymbol{k})^{-1} \leq \frac{1}{\sqrt{\left(\frac{\pi}{L}\right)^{2}(d-1)+\left(\frac{\pi}{L_{d}}\right)^{2}}}, \quad \boldsymbol{k} \in \Gamma .
$$

Hence $h^{-1} \in \mathfrak{B}\left(L^{2}(\Lambda)\right)$.

We set

$$
L_{\text {real }}^{2}(\Lambda):=\left\{f \in L^{2}(\Lambda) \mid f^{*}=f\right\},
$$

the real Hilbert space of real elements in $L^{2}(\Lambda)$. For all $\alpha \in \mathbb{R}$ and $f \in D\left(h^{\alpha}\right)$, we have

$$
h^{\alpha} f=\sum_{\boldsymbol{k} \in \Gamma} \omega(\boldsymbol{k})^{\alpha}\left\langle\varphi_{\boldsymbol{k}}, f\right\rangle \varphi_{\boldsymbol{k}}
$$

in the sense of $L^{2}(\Lambda)$-convergence. Hence

$$
h^{\alpha} D\left(h^{\alpha}\right) \cap L_{\text {real }}(\Lambda) \subset L_{\text {real }}^{2}(\Lambda) .
$$

We next consider the case where a "wall"

$$
W_{a}:=\left\{\boldsymbol{x} \in \Lambda \mid x_{d}=a\right\}
$$


perpendicular to the $x_{d}$-axis is placed in $\Lambda$ with $0<a<L_{d}$. In this case $\Lambda$ is decomposed as follows:

$$
\Lambda=\Lambda_{1} \cup W_{a} \cup \Lambda_{2}
$$

where

$$
\Lambda_{1}:=\left\{\boldsymbol{x} \in \Lambda \mid 0<x_{d}<a\right\}, \quad \Lambda_{2}:=\left\{\boldsymbol{x} \in \Lambda \mid a<x_{d}<L_{d}\right\} .
$$

This decomposition of the box $\Lambda$ induces the orthogonal decomposition

$$
L^{2}(\Lambda)=L^{2}\left(\Lambda_{1}\right) \oplus L^{2}\left(\Lambda_{2}\right)
$$

of the Hilbert space $L^{2}(\Lambda)$. But one should note that the Dirichlet Laplacian $\Delta_{\mathrm{D}}$ cannot be reduced by $L^{2}\left(\Lambda_{\ell}\right)(\ell=1,2)$.

In what follows, we assume the following:

Assumption (a) $L^{2} / L_{d}^{2} \in \mathbb{Q}$ (the set of rational numbers) and $a^{2} / L_{d}^{2} \notin \mathbb{Q}$

Let $\Lambda_{1}$ and $\Lambda_{2}$ be given by (6.13) and $\Delta_{\ell}$ be the Dirichlet Laplacian for $\Lambda_{\ell}(\ell=1,2)$. For each $\boldsymbol{k} \in \Gamma$ and $\ell=1,2$, we define functions $\psi_{\boldsymbol{k}}^{(\ell)}$ on $\Lambda_{\ell}$ as follows:

$$
\begin{aligned}
& \psi_{\boldsymbol{k}}^{(1)}(\boldsymbol{x}):=\left(\prod_{j=1}^{d-1} \varphi_{k_{j}}\left(x_{j}\right)\right) \psi_{k_{d}}^{(1)}\left(x_{d}\right), \quad \boldsymbol{x} \in \Lambda_{1}, \\
& \psi_{\boldsymbol{k}}^{(2)}(\boldsymbol{x}):=\left(\prod_{j=1}^{d-1} \varphi_{k_{j}}\left(x_{j}\right)\right) \psi_{k_{d}}^{(2)}\left(x_{d}\right), \quad \boldsymbol{x} \in \Lambda_{2},
\end{aligned}
$$

where

$$
\begin{aligned}
\psi_{k_{d}}^{(1)}\left(x_{d}\right) & :=\sqrt{\frac{2}{a} \sin \frac{L_{d} k_{d} x_{d}}{a}, \quad x_{d} \in(0, a),} \\
\psi_{k_{d}}^{(2)}\left(x_{d}\right) & :=\sqrt{\frac{2}{L-a}} \sin \frac{L_{d} k_{d}\left(x_{d}-a\right)}{L_{d}-a}, \quad x_{d} \in\left(a, L_{d}\right) .
\end{aligned}
$$

The set $\left\{\psi_{\boldsymbol{k}}^{(\ell)}\right\}_{\boldsymbol{k} \in \Gamma}$ is a CONS of $L^{2}\left(\Lambda_{\ell}\right)$.

Using the Dirichlet Laplacian $\Delta_{\ell}$ for $\Lambda_{\ell}(\ell=1,2)$, we introduce a non-negative selfadjoint operator

$$
h_{a, \ell}:=\left(-\Delta_{\ell}+m^{2}\right)^{1 / 2}
$$

on $L^{2}\left(\Lambda_{\ell}\right)$ and define

$$
h_{a}:=h_{a, 1} \oplus h_{a, 2}
$$

relative to the decomposition (6.14). 
Let

$$
\begin{aligned}
\omega_{1}(\boldsymbol{k}) & :=\sqrt{k_{1}^{2}+\cdots+k_{d-1}^{2}+\left(L_{d} k_{d} / a\right)^{2}+m^{2}}, \\
\omega_{2}(\boldsymbol{k}) & :=\sqrt{k_{1}^{2}+\cdots+k_{d-1}^{2}+\left(L_{d} k_{d} /\left(L_{d}-a\right)\right)^{2}+m^{2}}, \quad \boldsymbol{k} \in \Gamma .
\end{aligned}
$$

Then we have

$$
h_{a, \ell} \psi_{\boldsymbol{k}}^{(\ell)}=\omega_{\ell}(\boldsymbol{k}) \psi_{\boldsymbol{k}}^{(\ell)}, \quad \boldsymbol{k} \in \Gamma .
$$

By (6.14), each $f \in L^{2}(\Lambda)$ is written as

$$
f=f^{(1)}+f^{(2)}
$$

or $f=\left(f^{(1)}, f^{(2)}\right)$, where

$$
f^{(\ell)}:=\chi_{\Lambda_{\ell}} f \in L^{2}\left(\Lambda_{\ell}\right)
$$

with $\chi_{\Lambda_{\ell}}$ being the characteristic function of $\Lambda_{\ell}$.

The direct sum operator

$$
-\Delta_{12}:=\left(-\Delta_{1}\right) \oplus\left(-\Delta_{2}\right)
$$

is a non-negative self-adjoint operator on $L^{2}(\Lambda)$. By functional calculus, we have

$$
\left(-\Delta_{12}\right)^{1 / 2}=\left(-\Delta_{1}\right)^{1 / 2} \oplus\left(-\Delta_{2}\right)^{1 / 2} .
$$

Lemma 6.2 $D\left(\left(-\Delta_{12}\right)^{1 / 2}\right) \subset D\left(\left(-\Delta_{\mathrm{D}}\right)^{1 / 2}\right)$ and, for all $f \in D\left(\left(-\Delta_{12}\right)^{1 / 2}\right)$,

$$
\left\|\left(-\Delta_{\mathrm{D}}\right)^{1 / 2} f\right\|=\left\|\left(-\Delta_{12}\right)^{1 / 2} f\right\| .
$$

In particular, $\left(-\Delta_{\mathrm{D}}\right)^{1 / 2}\left(-\Delta_{12}\right)^{-1 / 2}$ is in $\mathfrak{B}\left(L^{2}(\Lambda)\right)$.

Proof. Let $f=f^{(1)}+f^{(2)} \in D\left(\left(-\Delta_{12}\right)^{1 / 2}\right)$. Then, by the definition of $\Delta_{\ell}$, there exists a sequence $\left\{f_{n}^{(\ell)}\right\}_{n=1}^{\infty}$ in $C_{0}^{\infty}\left(\Lambda_{\ell}\right)$ such that $f_{n}^{(\ell)} \rightarrow f^{(\ell)}(n \rightarrow \infty)$ and

$$
\left\|\left(-\Delta_{\ell}\right)^{1 / 2} f^{(\ell)}\right\|^{2}=\lim _{n \rightarrow \infty} \sum_{j=1}^{d}\left\|\partial_{j} f_{n}^{(\ell)}\right\|^{2}
$$

where $\partial_{j}:=\partial / \partial x_{j}$. Hence, letting $f_{n}:=f_{n}^{(1)}+f_{n}^{(2)}$, we see that $f_{n} \in C_{0}^{\infty}(\Lambda)$ and $f_{n} \rightarrow f$ $(n \rightarrow \infty)$,

$$
\lim _{n \rightarrow \infty} \sum_{j=1}^{d}\left\|\partial_{j} f_{n}\right\|^{2}=\left\|\left(-\Delta_{1}\right)^{1 / 2} f^{(1)}\right\|^{2}+\left\|\left(-\Delta_{2}\right)^{1 / 2} f^{(2)}\right\|^{2}=\left\|\left(-\Delta_{12}\right)^{1 / 2} f\right\|^{2} .
$$

Therefore $f \in D\left(\left(-\Delta_{\mathrm{D}}\right)^{1 / 2}\right)$ (hence $\left.D\left(\left(-\Delta_{12}\right)^{1 / 2}\right) \subset D\left(\left(-\Delta_{\mathrm{D}}\right)^{1 / 2}\right)\right)$ and $(6.23)$ holds.

We next study relations between $h$ and $h_{a}$.

\section{Lemma 6.3}


(i) $D\left(h_{a}\right) \subset D(h)$ and $h h_{a}^{-1}$ is in $\mathfrak{B}\left(L^{2}(\Lambda)\right)$.

(ii) $D\left(h_{a}^{1 / 2}\right) \subset D\left(h^{1 / 2}\right)$ and $h^{1 / 2} h_{a}^{-1 / 2}$ is in $\mathfrak{B}\left(L^{2}(\Lambda)\right)$.

Proof. (i) It follows from functional calculus that

$$
D(h)=D\left(\left(-\Delta_{\mathrm{D}}\right)^{1 / 2}\right), \quad D\left(h_{a}\right)=D\left(\left(-\Delta_{12}\right)^{1 / 2}\right) .
$$

Hence, by Lemma $6.2, D\left(h_{a}\right) \subset D(h)$. Hence $D\left(h h_{a}^{-1}\right)=L^{2}(\Lambda)$ and $h h_{a}^{-1} \in \mathfrak{B}\left(L^{2}(\Lambda)\right){ }^{3}$

(ii) By part (i), $h$ is $h_{a}$-bounded. Since $h$ and $h_{a}$ are non-negative self-adjoint operators, it follows from a general theorem ([8, Theorem X.18(a)]) that $h^{1 / 2}$ is $h_{a}^{1 / 2}$-bounded. Hence $D\left(h_{a}^{1 / 2}\right) \subset D\left(h^{1 / 2}\right)$ and $h^{1 / 2} h_{a}^{-1 / 2}$ is bounded with $D\left(h^{1 / 2} h_{a}^{-1 / 2}\right)=L^{2}(\Lambda)$.

For convenience, we extend each eigenfunction $\psi_{\boldsymbol{k}}^{(\ell)}$ of $h_{a, \ell}(\boldsymbol{k} \in \Gamma)$ to a function on $\Lambda$ in the following way:

$$
\tilde{\psi}_{\boldsymbol{k}}^{(\ell)}(\boldsymbol{x}):=\left\{\begin{array}{cc}
\psi_{\boldsymbol{k}}^{(\ell)}(\boldsymbol{x}) & \text { if } \boldsymbol{x} \in \Lambda_{\ell} \\
0 & \text { if } \boldsymbol{x} \in \Lambda \backslash \Lambda_{\ell}
\end{array} .\right.
$$

Similarly we denote by $\tilde{\psi}_{k_{d}}^{(\ell)}(\ell=1,2)$ the extensions of the functions $\psi_{k_{d}}^{(1)}$ and $\psi_{k_{d}}^{(2)}$ to $\left(0, L_{d}\right)$.

By functional calculus, we have for all $\alpha>0$

$$
h_{a}^{\alpha} \tilde{\psi}_{\boldsymbol{k}}^{(\ell)}=\omega_{\ell}(\boldsymbol{k})^{\alpha} \tilde{\psi}_{\boldsymbol{k}}^{(\ell)}, \quad \ell=1,2, \boldsymbol{k} \in \Gamma .
$$

For each $(\boldsymbol{k}, \boldsymbol{p}) \in \Gamma \times \Gamma$ and $\ell=1,2$, we define

$$
\gamma_{\boldsymbol{k} \boldsymbol{p}}^{(\ell)}:=\left\langle\varphi_{\boldsymbol{k}}, \tilde{\psi}_{\boldsymbol{p}}^{(\ell)}\right\rangle
$$

It follows from (6.2), (6.15) and (6.16) that

$$
\gamma_{\boldsymbol{k} \boldsymbol{p}}^{(\ell)}=\left(\prod_{j=1}^{d-1} \delta_{k_{j} p_{j}}\right)\left\langle\varphi_{k_{d}}, \tilde{\psi}_{p_{d}}^{(\ell)}\right\rangle .
$$

We set

$$
c_{1}:=\frac{L_{d}}{a}, \quad c_{2}:=\frac{L_{d}}{L_{d}-a} .
$$

Lemma 6.4 For all $k_{d}, p_{d} \in\left(\pi / L_{d}\right) \mathbb{N}$,

$$
\begin{aligned}
\left\langle\varphi_{k_{d}}, \tilde{\psi}_{p_{d}}^{(1)}\right\rangle & =\frac{2}{\sqrt{L_{d} a}} \frac{(-1)^{L_{d} p_{d}} c_{1} p_{d} \sin \left(a k_{d}\right)}{k_{d}^{2}-c_{1}^{2} p_{d}^{2}}, \\
\left\langle\varphi_{k_{d}}, \tilde{\psi}_{p_{d}}^{(2)}\right\rangle & =-\frac{2}{\sqrt{L_{d}\left(L_{d}-a\right)}} \frac{c_{2} p_{d} \sin \left(a k_{d}\right)}{k_{d}^{2}-c_{2}^{2} p_{d}^{2}} .
\end{aligned}
$$

\footnotetext{
${ }^{3}$ The following fact is well known (which follows from the closed graph theorem): let $A$ and $B$ be closed linear operators on a Banach space $\mathscr{X}$. Suppose that $D(A) \subset D(B)$ and $A$ is bijective. Then $B A^{-1}$ is in $\mathfrak{B}(\mathscr{X})$.
} 
Proof. By (6.4) and (6.17), we have

$$
\left\langle\varphi_{k_{d}}, \tilde{\psi}_{p_{d}}^{(1)}\right\rangle=\frac{2}{\sqrt{L_{d} a}} \int_{0}^{a} \sin \left(k_{d} x_{d}\right) \sin \left(c_{1} p_{d} x_{d}\right) d x_{d} .
$$

Then, by direct computations, we obtain (6.29). Similarly one can prove (6.30).

Lemma 6.5 $D\left(h_{a}^{1 / 2}\right) \varsubsetneqq D\left(h^{1 / 2}\right)$.

Proof. By functional calculus, for all $\boldsymbol{k} \in \Gamma, \varphi_{\boldsymbol{k}} \in D\left(h^{1 / 2}\right)$ with

$$
h^{1 / 2} \varphi_{\boldsymbol{k}}=\omega(\boldsymbol{k})^{1 / 2} \varphi_{\boldsymbol{k}}
$$

It is sufficient to show that, for some $\boldsymbol{k}_{0} \in \Gamma, \varphi_{\boldsymbol{k}_{0}} \notin D\left(h_{a}^{1 / 2}\right)$. Let $\boldsymbol{k} \in \Gamma$ be such that $\varphi_{\boldsymbol{k}} \in D\left(h_{a}^{1 / 2}\right)$. Then, by the fact that $\left\{\tilde{\psi}_{\boldsymbol{p}}^{(\ell)} \mid \ell=1,2, \boldsymbol{p} \in \Gamma\right\}$ is a CONS of $L^{2}(\Lambda)$, we have

$$
s_{\boldsymbol{k}}:=\sum_{\ell=1}^{2} \sum_{\boldsymbol{p} \in \Gamma}\left|\left\langle\tilde{\psi}_{\boldsymbol{p}}^{(\ell)}, h_{a}^{1 / 2} \varphi_{\boldsymbol{k}}\right\rangle\right|^{2}=\left\|h_{a}^{1 / 2} \varphi_{\boldsymbol{k}}\right\|^{2}<\infty
$$

By the symmetry of $h_{a}^{1 / 2}$ and (6.25), we have

$$
\left\langle\tilde{\psi}_{\boldsymbol{p}}^{(\ell)}, h_{a}^{1 / 2} \varphi_{\boldsymbol{k}}\right\rangle=\left\langle h_{a}^{1 / 2} \tilde{\psi}_{\boldsymbol{p}}^{(\ell)}, \varphi_{\boldsymbol{k}}\right\rangle=\omega_{\ell}(\boldsymbol{p})^{1 / 2} \gamma_{\boldsymbol{k} \boldsymbol{p}}^{(\ell)}
$$

Hence

$$
s_{\boldsymbol{k}}=\sum_{\ell=1}^{2} \sum_{\boldsymbol{p} \in \Gamma} \omega_{\ell}(\boldsymbol{p})\left|\gamma_{\boldsymbol{k} \boldsymbol{p}}^{(\ell)}\right|^{2}
$$

Using (6.27), we have

$$
s_{\boldsymbol{k}}=\sum_{\ell=1}^{2} \sum_{p_{d} \in\left(\pi / L_{d}\right) \mathbb{N}} \omega_{\ell}\left(\tilde{\boldsymbol{k}}, p_{d}\right)\left|\left\langle\varphi_{k_{d}}, \tilde{\psi}_{p_{d}}^{(\ell)}\right\rangle\right|^{2}
$$

where

$$
\tilde{\boldsymbol{k}}:=\left(k_{1}, \ldots, k_{d-1}\right) \in\left(\frac{\pi}{L} \mathbb{N}\right)^{d-1}
$$

By Lemma 6.4, we have

$$
\lim _{p_{d} \rightarrow \infty}\left|p_{d}\left\langle\varphi_{k_{d}}, \tilde{\psi}_{p_{d}}^{(1)}\right\rangle\right|^{2}=\frac{4 \sin ^{2}\left(a k_{d}\right)}{L_{d} a c_{1}^{2}} . \quad \lim _{p_{d} \rightarrow \infty}\left|p_{d}\left\langle\varphi_{k_{d}}, \tilde{\psi}_{p_{d}}^{(2)}\right\rangle\right|^{2}=\frac{4 \sin ^{2}\left(a k_{d}\right)}{L_{d}\left(L_{d}-a\right) c_{2}^{2}} .
$$

Now let $\boldsymbol{k}_{0}:=\left(\tilde{\boldsymbol{k}}, k_{d}\right)$ with $\tilde{\boldsymbol{k}} \in((\pi / L) \mathbb{N})^{d-1}$ arbitrary and $k_{d}=\pi / L_{d}$. Then $0<a k_{d}<\pi$. Hence $\sin a k_{d} \neq 0$. Therefore there exists a constant $C>0$ such that

$$
\left|\left\langle\varphi_{k_{d}}, \tilde{\psi}_{p_{d}}^{(\ell)}\right\rangle\right|^{2} \geq \frac{C}{c_{\ell} p_{d}^{2}} .
$$


Note that

$$
\omega_{\ell}\left(\tilde{\boldsymbol{k}}, p_{d}\right) \geq c_{\ell} p_{d}
$$

Hence

$$
s_{\boldsymbol{k}_{0}} \geq C \sum_{p_{d} \in\left(\pi / L_{d}\right) \mathbb{N}} \frac{1}{p_{d}}=\infty .
$$

Thus $\varphi_{\boldsymbol{k}_{0}} \notin D\left(h_{a}^{1 / 2}\right)$.

The following fact shows a singular nature of the pair $\left(h, h_{a}\right)$ of one-particle Hamiltonians:

Lemma 6.6 The operator $h^{-1 / 2} h_{a}^{1 / 2}$ is unbounded.

Proof. Let $T:=h^{-1 / 2} h_{a}^{1 / 2}$. We prove the unboundedness of $T$ by reductio ad absurdum. Suppose that $T$ were bounded. Since $D(T)=D\left(h_{a}^{1 / 2}\right), T$ is densely defined. Hence it is closable and the closure $\bar{T}$ is in $\mathfrak{B}\left(L^{2}(\Lambda)\right)$. We have $T^{*}=(\bar{T})^{*}$. Hence $D\left(T^{*}\right)=L^{2}(\Lambda)$. Since $h^{-1 / 2}$ is bounded with $D\left(h^{-1 / 2}\right)=L^{2}(\Lambda)$, it follows that $T^{*}=\left(h_{a}^{1 / 2}\right)^{*}\left(h^{-1 / 2}\right)^{*}=$ $h_{a}^{1 / 2} h^{-1 / 2}$. This implies that $D\left(h^{1 / 2}\right) \subset D\left(h_{a}^{1 / 2}\right)$ and $D\left(T^{*}\right)=h^{1 / 2} D\left(h_{a}^{1 / 2}\right)$. But this contradicts Lemma 6.5.

Lemma 6.7 Let

$$
S_{ \pm}:=\frac{1}{2}\left(h^{-1 / 2} h_{a}^{1 / 2} \pm h^{1 / 2} h_{a}^{-1 / 2}\right)
$$

Then $S_{ \pm}$are unbounded.

Proof. This follows from Lemma 6.3(ii) and Lemma 6.6.

Note that

$$
\mathscr{D}_{a}:=D\left(S_{+}\right)=D\left(S_{-}\right)=D\left(h_{a}^{1 / 2}\right) .
$$

We denote by $C_{\Lambda}$ the complex conjugation on $L^{2}(\Lambda)$ :

$$
C_{\Lambda} f:=f^{*}, \quad f \in L^{2}(\Lambda) .
$$

Then it is easy to see that, for all $\alpha \in \mathbb{R}$,

$$
C_{\Lambda} h^{\alpha} \subset h^{\alpha} C_{\Lambda}, \quad C_{\Lambda} h_{a}^{\alpha} \subset h_{a}^{\alpha} C_{\Lambda}
$$

Hence

$$
C_{\Lambda} S_{ \pm} \subset S_{ \pm} C_{\Lambda}
$$

The next lemma follows from Lemma 5.1 and (6.33): 
Lemma 6.8 For all $f, g \in \mathscr{D}_{a}$, the following equations hold:

$$
\begin{aligned}
& \left\langle S_{+} f, S_{+} g\right\rangle-\left\langle S_{-} f, S_{-} g\right\rangle=\langle f, g\rangle, \\
& \left\langle S_{+} f, S_{-} g\right\rangle=\left\langle S_{-} f, S_{+} g\right\rangle .
\end{aligned}
$$

Lemma 6.9 The range $\operatorname{Ran} S_{+}$of $S_{+}$is dense in $L^{2}(\Lambda)$.

Proof. It is sufficient to show that the orthogonal complement $\left(\operatorname{Ran} S_{+}\right)^{\perp}$ of $\operatorname{Ran} S_{+}$ is $\{0\}$. Let $g \in\left(\operatorname{Ran} S_{+}\right)^{\perp}$. Then, for all $f \in D\left(h_{a}^{1 / 2}\right),\left\langle S_{+} f, g\right\rangle=0$, which implies that $\left\langle h_{a}^{1 / 2} f, h^{-1 / 2} g\right\rangle=-\langle G f, g\rangle$ with $G:=h^{1 / 2} h_{a}^{-1 / 2}$. By Lemma 6.3(ii), $G$ is bounded. Hence $\langle G f, g\rangle=\left\langle f, G^{*} g\right\rangle$. Hence $h^{-1 / 2} g \in D\left(h_{a}^{1 / 2}\right)$ and $h_{a}^{1 / 2} h^{-1 / 2} g=-G^{*} g$. Hence $g=-G G^{*} g$, which implies that $\|g\|^{2}=-\left\|G^{*} g\right\|^{2} \leq 0$. Therefore $g=0$. Thus $\left(\operatorname{Ran} S_{+}\right)^{\perp}=\{0\}$.

Lemma $6.10\left(h^{-1 / 2} h_{a}^{1 / 2}+i h^{1 / 2} h_{a}^{-1 / 2}\right)\left(\mathscr{D}_{a} \cap L_{\text {real }}^{2}(\Lambda)\right)$ is dense in $L^{2}(\Lambda)$.

Proof. Suppose that there exists an element $g \in L^{2}(\Lambda)$ such that, for all $f \in \mathscr{D}_{a} \cap$ $L_{\text {real }}(\Lambda),\left\langle g,\left(h^{-1 / 2} h_{a}^{1 / 2}+i h^{1 / 2} h_{a}^{-1 / 2}\right) f\right\rangle=0 \cdots(*)$. Since $C_{\Lambda} h_{a}^{1 / 2} \subset h_{a}^{1 / 2} C_{\Lambda}$, it follows that, each $f \in \mathscr{D}_{a}$ is written $f=f_{1}+i f_{2}$ with $f_{1}$ and $f_{2}$ being in $\mathscr{D}_{a} \cap L_{\text {real }}(\Lambda)$. Hence (*) extends to all $f \in \mathscr{D}_{a}$. Let $G:=h^{1 / 2} h_{a}^{-1 / 2}$. Then, by Lemma 6.3(ii), $G$ is bounded. Hence it follows that, for all $f \in \mathscr{D}_{a}=D\left(h_{a}^{1 / 2}\right),\left\langle h^{-1 / 2} g, h_{a}^{1 / 2} f\right\rangle=\left\langle i G^{*} g, f\right\rangle$. This implies that $h^{-1 / 2} g \in D\left(h_{a}^{1 / 2}\right)$ and $h_{a}^{1 / 2} h^{-1 / 2} g=i G^{*} g$. Hence $g=i G G^{*} g$, which implies that $\|g\|^{2}=i\left\|G^{*} g\right\|^{2}$. Hence $\|g\|^{2}=0$, implying that $g=0$. Thus the desired result follows.

We now define

$$
b(f):=\overline{a\left(S_{+} f\right)+a\left(C_{\Lambda} S_{-} f\right)^{*}}, \quad f \in \mathscr{D}_{a} .
$$

Theorem 6.11 The triple

$$
\pi_{b}\left(\mathscr{D}_{a}\right):=\left(\mathscr{F}_{\mathrm{b}}\left(L^{2}(\Lambda)\right), \mathscr{F}_{0}\left(L^{2}(\Lambda)\right),\left\{b(f), b(f)^{*} \mid f \in \mathscr{D}_{a}\right\}\right)
$$

is a representation of the CCRs over $\mathscr{D}_{a}$. Moreover, the following (i) and (ii) hold:

(i) There exists no non-zero vector $\Omega \in \cap_{f \in \mathscr{D}_{a}} D(b(f))$ such that $b(f) \Omega=0, f \in \mathscr{D}_{a}$.

(ii) The representation $\pi_{b}\left(\mathscr{D}_{a}\right)$ is irreducible and inequivalent to the Fock representation $\pi_{\mathrm{F}}\left(\mathscr{D}_{a}\right)$.

Proof. (i) By Lemma 6.9, we can apply Theorem 5.3(i) to the case where $K=h^{1 / 2}$, $L=h_{a}^{1 / 2}, J=C_{\Lambda}$ and $T_{+}=S_{+}$. Then the desired result follows.

(ii) The irreducibility of $\pi_{b}\left(\mathscr{D}_{a}\right)$ follows from Lemma 6.10 and an application of Theorem 5.4. The inequivalence of $\pi_{b}\left(\mathscr{D}_{a}\right)$ to $\pi_{\mathrm{F}}\left(\mathscr{D}_{a}\right)$ is due to an application of Theorem 5.3 (ii). 


\section{Acknowledgment}

The author thanks the referee for constructive comments on the original manuscript, which have been helpful in writing this revised version.

\section{References}

[1] A. Arai, A family of inequivalent Weyl representations of canonical commutation relations with applications to quantum field theory. Rev. Math. Phys. 28 (2016), 1650007 (26 pages).

[2] A. Arai, Analysis on Fock Spaces and Mathematical Theory of Quantum Fields, World Scientific, Singapore, 2018.

[3] H. B. G. Casimir, On the attraction between two perfectly conducting plates, Proc. Koninklijke Nederlandse Akademie van Wetenschappen 51 (1948), 793-795.

[4] C. Dappiaggi, G. Nosari and N. Pinamonti, The Casimir effect from the point of view of algebraic quantum field theory, Math. Phys. Anal. Geom.19 (2016), 12 (44 pages).

[5] H. Ezawa, K. Nakamura and K. Watanabe, The Casimir force from Lorentz's, in Frontiers in Quantum Physics (S. C. Lim, R. Abd-Shukor, K. H. Kwek eds.), Springer, Singapore, 1998, 160-169.

[6] F. Hiroshima, I. Sasaki, H. Spohn and A. Suzuki, Enhanced Binding in Quantum Field Theory, COE Lecture Note Vol. 38, Institute of Mathematics for Industry, Kyushu University, 2012.

[7] S. K. Lamoreaux, Demonstration of the Casimir force in the 0.6 to $6 \mu \mathrm{m}$ range, Phys. Rev. Lett. 78 (1998), 5-8; Erratum Phys. Rev. Lett. 81 (1998), 5475-5476.

[8] M. Reed and B. Simon, Methods of Modern Mathematical Physics II: Fourier Analysis, Self-Adjointness, Academic Press, New York, 1975.

[9] M. Reed and B. Simon, Methods of Modern Mathematical Physics III: Scattering Theory, Academic Press, New York, 1979.

[10] M. Reed and B. Simon, Methods of Modern Mathematical Physics IV: Analysis of Operators, Academic Press, Neew York, 1978.

[11] S. N. M. Ruijsenaars, On Bogoliubov transformations for systems of relativistic charged particles, J. Math. Phys. 18 (1977), 517-526

[12] S. N. M. Ruijsenaars, On Bogoliubov transformations. II. The general case, Ann. of Phys. 116 (1978), 105-134.

[13] D. Shale, Linear symmetries of free boson fields, Trans. Amer. Math. Soc. 103 (1962), 149-167. 
[14] M. J. Sparnaay, Measurements of attractive forces between flat plates, Physica 24 (1958), 751-764. 